\title{
An ultrasound observation study on the levator hiatus with or without diastasis recti abdominis in postpartum women
}

\author{
Peng Tian ${ }^{1,2} \cdot$ Dong Mei Liu ${ }^{1} \cdot$ Chao Wang ${ }^{1} \cdot \mathrm{Yu} \mathrm{Gu}^{1} \cdot$ Guo Qing Du ${ }^{1} \cdot$ Jia Wei Tian ${ }^{1}$
}

Received: 13 December 2020 / Accepted: 24 March 2021 / Published online: 17 April 2021

(C) The Author(s) 2021

\begin{abstract}
Introduction and hypothesis We hypothesized that differences in post-partum levator hiatus (LH) measurements, as well as the area of urethra and bladder (AUB), viewed under ultrasound, correlate with diastasis rectus abdominis (DRA) occurrence. The primary objective of this study is to determine ultrasound parameters available for diagnosing DRA in post-partum women. We compared LH and AUB measurements under ultrasound in primiparous women, with and without DRA, at 24-26 weeks postpartum.

Methods One hundred ninety-four women underwent routine examination, including a self-made clinical symptoms questionnaire, DRA evaluation, and LH and AUB measurements. Independent samples $t$ - and chi-squared tests were used to compare the differences between women with and without DRA.

Results DRA incidence was significantly higher among those who underwent cesarean section (CS) than for vaginal delivery (VD) $(P=0.038)$. DRA patients could potentially have urinary urgency, frequency, pain, dysuria, and perineal tears. Additionally, statistically significant differences were found between VD patients, with or without DRA, in the resting LH transverse diameter $(\mathrm{TrD})(P=0.032)$ and the area of the levator hiatus (ALH) $(P=0.048)$ as well as AUB at Valsalva $(P=$ 0.049). No differences, however, were found between the DRA and no DRA groups for all those measurements among women who had cesarean deliveries.

Conclusions DRA was more likely in post-CS women. Furthermore, the results showed a plausible association between DRA occurrence and LH expansion, especially in women with VD under rest and Valsalva. This could be useful for developing therapeutic plans based on these parameters for post-partum rehabilitation of women with DRA to avoid long-term complications.
\end{abstract}

Keywords Diastasis recti abdominis $\cdot$ Levator hiatus $\cdot$ Mode of delivery $\cdot$ Ultrasound

\section{Introduction}

The rectus abdominus is involved in stabilizing an individual's trunk and pelvis, helping to maintain posture and

Peng Tian and Dong Mei Liu contributed equally to this work.

Guo Qing Du

duguoqing9@163.com

$\triangle$ Jia Wei Tian

jwtian2004@163.com

1 Department of Ultrasonography, The Second Affiliated Hospital of Harbin Medical University, No. 246, Xuefu Road, Nanggang District Harbin 150081, People's Republic of China

2 Department of Ultrasonography, The Daqing Oilfield General Hospital, Daqing, People's Republic of China enable them to participate in regular physical activity. Diastasis rectus abdominis (DRA) is the increase in the inner diameter of the rectus abdominis on both sides of the linea alba, which commonly results in twisted/ abnormal postures [1]. DRA can possibly lead to limitations in conducting physical exercise, as well as lumbosacral and hip pain, ultimately negatively impacting the individual's quality of life [2]. Pregnancy and delivery have been regarded as major risk factors for DRA and pelvic floor dysfunction (PFD), which is supported by a study demonstrating a relationship between DRA and support-related pelvic floor dysfunction diagnoses of stress urinary incontinence (UI), fecal incontinence, and pelvic organ prolapse (POP). However, such impacts have been contested, with some studies, such as one from Norway, finding that women with diastasis were no more 
likely to have weaker pelvic floor muscle strength (PFMS), UI, or POP. This finding is confirmed by research showing similar results in women at 6-8 weeks postpartum [3]. Another study likewise indicated that no significant difference was present between DRA and nonDRA groups with respect to lumbosacral pain $[4,5]$. In fact, the exact relation among pregnancy, delivery, DRA, and PFD is still a controversial topic among urologists, gynecologists, and obstetricians, and some studies have found either positive or negative correlations among those factors [1].

The inner diameter of the rectus abdominis and measurement of the levator hiatus (LH) can both be easily obtained using ultrasound, a simple, highly reliable, and non-invasive method [6-8]. Vaginal delivery is strongly associated with a larger, more distensible LH and a greater degree of bladder neck mobility, both antenatally and postpartum. Cesarean section reduces postnatal symptoms but does not prevent them [9]. Due to the contradictions in current studies regarding the relation of DRA, PFD, and related symptoms, we explored whether DRA was associated with LH to clarify the relationship between intra-abdominal and pelvic pressures.

\section{Materials and methods}

\section{Study subjects and determining sample size}

Ethical approval was not required in this study, because the data originated from the ultrasound report management system. All post-delivery patient examinations were routine ones carried out in our hospital [10]. After delivery, women were reviewed at 42 days, 3 months, and 6 months. Gynecological examination, as well as routine blood, urine, liver, and kidney function tests, plus ultrasonography, were performed as routine examinations. This was a cross-sectional study, and data were collected from the postpartum review clinic at the Daqing Oilfield General Hospital in China from January 2018 to February 2020.

For this analysis, the inclusion criteria were as follows (Fig. 1): Women who underwent regular examination at the maternity clinic during pregnancy and gave birth in Daqing Oilfield General Hospital, women coming back for examinations during 24-26 weeks postpartum, women with only one childbirth, women with complete obstetric data, women with body mass index $(\mathrm{BMI}) \leq 30 \mathrm{~kg} / \mathrm{m}^{2}$, and women between the ages of 20-45 years. Four hundred eighty-three women were admitted to our hospital for postpartum follow-up during this period. Two hundred eighty-nine women were excluded because they did not come to the hospital for a 24-26 week postpartum follow-up $(n=41)$, had POP $(n=40)$, had moved incorrectly during ultrasound, yielding unclear images $(n=$ 42 , meaning the women did not perform the Valsalva and contraction maneuver [11]), had incomplete obstetric/ postpartum records $(n=39)$, had delivered $>1$ baby $(n=$ $81)$, had a BMI $>30 \mathrm{~kg} / \mathrm{m}^{2}(n=31)$, or had a serious illness $(n=15)$. Statistical analyses were then performed on the remaining 194 women. The validity of using data from 194 cases in this study is confirmed via PASS software (version $15.0 ;(\alpha=0.05, \beta=0.20$, bilateral testing), utilizing previously published formulas, estimating that a suitable sample size for this study would be 104 [12-14].

A self-made questionnaire was designed to record postpartum females' age at birth, body weight $(\mathrm{kg})$ and height $(\mathrm{cm})$ at

Fig. 1 Flowchart

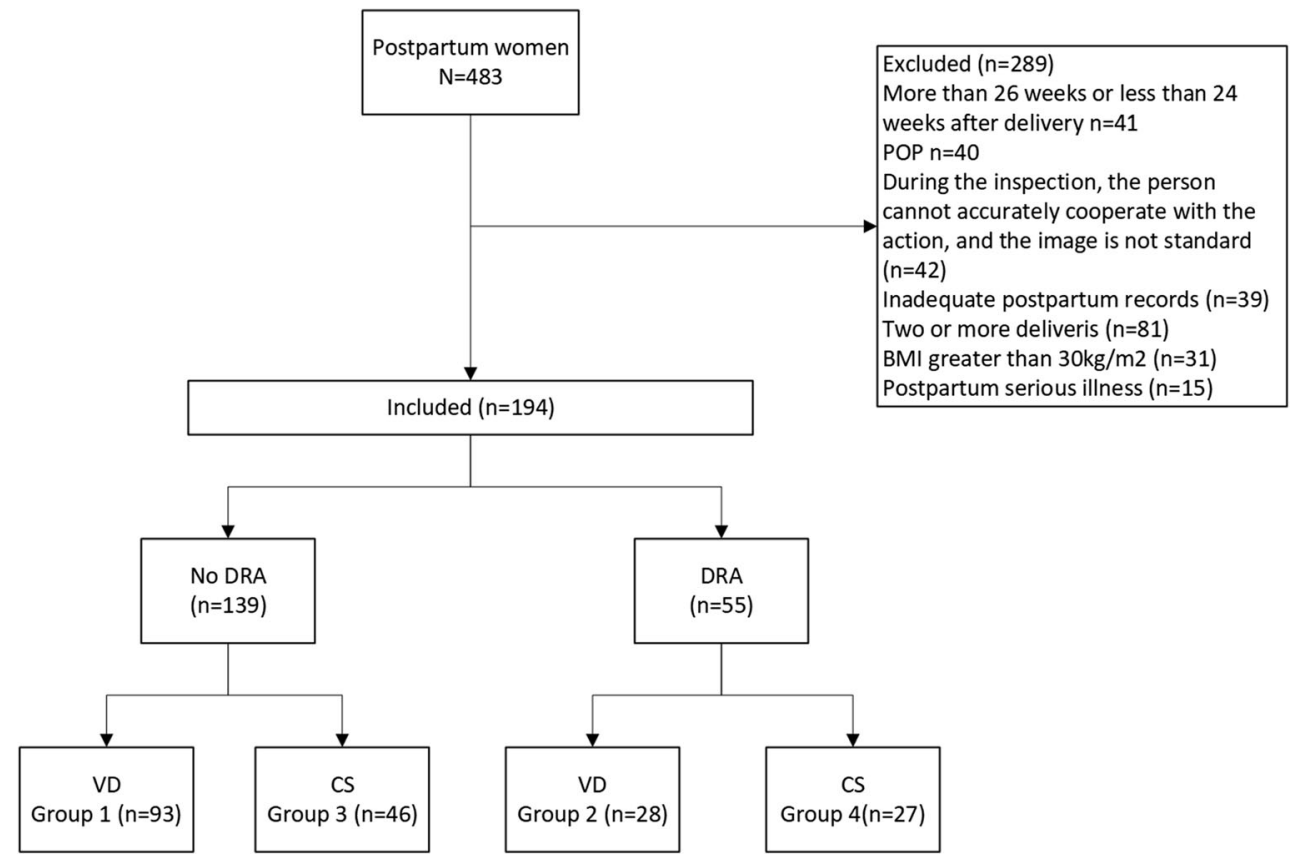


24-26 weeks postpartum, delivery mode, presence of lateral episiotomy or perineal tear, and the degree of urinary frequency, urgency, leakage, or dysuria. Two sonographers with respectively 12 and 20 years of ultrasound imaging experience performed all ultrasound imaging. The investigators were blinded to the participants' identification and delivery mode. The questionnaire study was completed by the physician assistant.

\section{Measurement standards and data recording}

A GE Voluson E8 ultrasound diagnostic device (GE Healthcare, Waukesha, WI), with a line array probe (4$12 \mathrm{MHz}$ frequency) was used to measure the rectus abdominis inner diameter, except when such a diameter was too wide. In those cases, a two-dimensional probe $(3.5-7.0 \mathrm{MHz})$ was utilized instead. A four-dimensional ultrasound probe (RAB4-8D with 5-10 MHz frequency) was chosen to measure the $\mathrm{LH}$ as well as the angle of urethra and bladder (AUB).

\section{Inter-rectus distance (IRD) measurement}

Participants were placed in a supine resting position (knees bent $90^{\circ}$, feet resting on the plinth, arms alongside the body) $[6,15-17]$. They were asked to perform abdominal contractions until the shoulder blades left the examination bed. The ultrasound transducer was placed transversely at three locations along the midline of the abdomen, using the center of the umbilicus as a reference: $2 \mathrm{~cm}$ below the umbilicus and 2 and $5 \mathrm{~cm}$ above the umbilicus (Fig. 2). To standardize the transducer position, each measurement location was marked with ink on the skin. All measurements were repeated three times, and the mean of the three resulting values was used. DRA diagnostic criteria are indicated below, where it was defined as a width $\geq 21 \mathrm{~mm}$ at $2 \mathrm{~cm}$ below the umbilicus, $\geq 28 \mathrm{~mm}$ at $2 \mathrm{~cm}$ above the umbilicus, or $\geq 24 \mathrm{~mm}$ at $5 \mathrm{~cm}$ above the umbilicus. Any values below those given above indicated no DRA was present in those patients [15].

We first divided the subjects into no-DRA $(n=139)$ and DRA groups $(n=55)$, according to the presence of DRA, as defined by the above parameters. The population was then further divided into four groups to further explore whether the delivery mode contributed to DRA's influence on the size of the LH or AUB: group I (vaginal delivery [VD] with no DRA, $n=93$ ), II (VD with DRA, $n=28$ ), III (cesarean section [CS] with no DRA, $n=46$ ), and IV (CS with DRA, $n=27$ ), as shown in Fig. 1. The size of each individual group was in accordance with the estimate determined using PASS software, in which each group would have no less than 26 members. This was based upon a $50 \%$ insurance cover level of the aforementioned estimated total sample size of 104 , in which a $50 \%$ sample size calculation was considered representative [18].

\section{Measurement of LH and AUB}

Bladder emptying was confirmed by transabdominal ultrasound by evaluating the post-void residual volume, where a pathological volume was defined as $>150 \mathrm{ml}$ urine. The convex volumetric ultrasound transducer was positioned translabially in the midsagittal plane, with patients in the lithotomy position. Measurements were performed in the axial plane of minimal hiatal dimensions $[19,20]$. The ultrasound technicians performing the examinations in this study demonstrated good intra- and inter-observer reliability, as well as consistency over multiple tests, for this procedure. Moreover, the results were comparable to ones obtained through different pelvic floor assessment techniques and diagnostic tools [21]. The antero-posterior diameter (A-PD), transverse diameter (TrD), and area of levator hiatus (ALH), as well as AUB, were recorded at rest, squeeze, and Valsalva maneuver (Supplementary Fig. 1). Three-dimensional volume acquisition was required for $\mathrm{ALH}, \mathrm{APD}$, and $\mathrm{TrD}$ measurements [19], while 2D acquisition was necessary for AUB [20] (Supplementary Fig. 2).

\section{Statistical analysis}

Statistical analysis was performed using IBM SPSS version 23.0. All parameters were measured three times, and the mean of those three measurements was used for analyses. Categorical variables are represented as numbers and
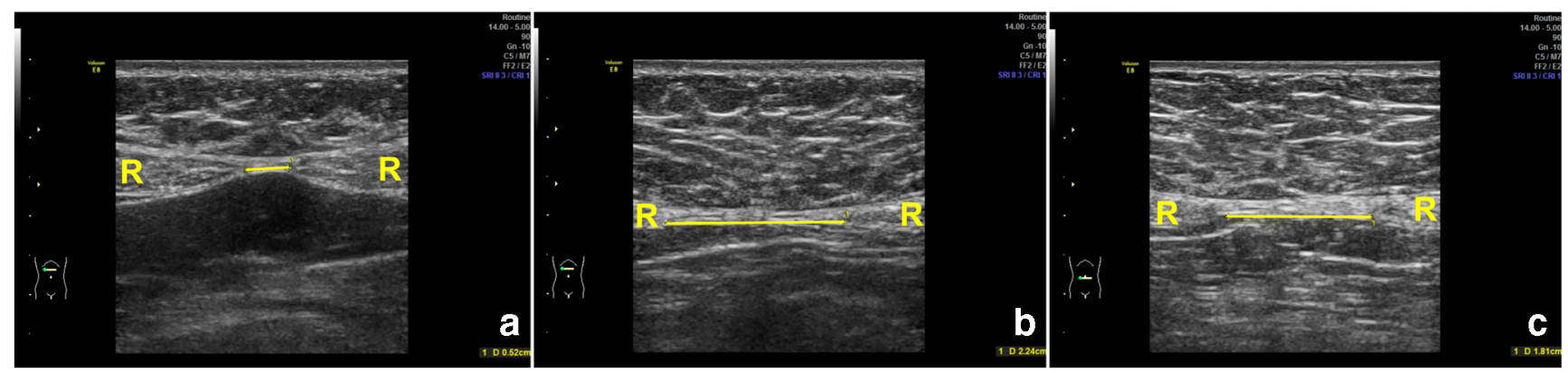

Fig. 2 Ultrasound images of the inter-rectus distance at $5 \mathrm{~cm}$ above (a), $2 \mathrm{~cm}$ above (b), and $2 \mathrm{~cm}$ below (c) the umbilicus. R: Rectus abdominis. Yellow line: Measurement between the two rectus abdominis muscles at the low edge of the connection 
percentages, while continuous ones are presented as mean \pm standard deviations (SDs) if the data follow a normal distribution. If a normal distribution was not followed by the data, medians (interquartile ranges) were calculated and utilized instead in data analysis. Chi-square tests were performed to compare the relationship between DRA and delivery mode as well as the prevalence of various clinical symptoms associated with DRA. Independent samples $t$-test was used to analyze differences in background characteristics between women with or without DRA under the circumstances of normally distributed data. $P<0.05$ was considered statistically significant.

\section{Results}

The overall prevalence of DRA in our samples was $28.4 \%$. No significant differences in maternal age, height, and BMI between women with and without DRA were found, though the DRA group had a slightly higher, but still statistically insignificant, body weight compared to those without DRA, at 64 and $60 \mathrm{~kg}$, respectively. However, with respect to different delivery modes, DRA incidence in the CS group was significantly higher than in the VD group $(P=0.038$; Table 1$)$.

Using our simple self-made questionnaire, we found that a significant difference was present with respect to the occurrence of perineal tear between the DRA and no-DRA groups, where it was more common in the former $(P=0.005$; Table 2$)$.

Table 3 shows the relationship between the different delivery modes and clinical symptom prevalence with respect to the above-mentioned groups I-IV. The difference with respect to perineal tear was still present even when delivery mode was taken into account, where it was more prevalent among group II (VD with DRA) than group I (VD, no DRA) $(P=0.000)$. Higher percentages were found for group IV (CS with DRA) compared to Group III (CS, no DRA) with respect to urinary frequency, being $25.9 \%$ for "occasional" and $3.7 \%$ for "usual" occurrences in group IV versus $8.7 \%$ and $0 \%$ respectively in
Table 2 Clinical symptoms in women with or without DRA

\begin{tabular}{llll}
$\begin{array}{l}\text { All } \\
\text { subjects }\end{array}$ & No DRA & DRA & $\begin{array}{l}P \\
\text { value }\end{array}$ \\
\hline
\end{tabular}

Perineal-lateral incision $(n$,

$\%)$

Yes

No

$54(27.8) \quad 42(30.2) \quad 12(21.8) \quad 0.240$

Perineal tear $(n, \%)$

Yes

No

$140(72.2) \quad 97(69.8) \quad 43(78.2)$

$15(7.7) \quad 6(4.3) \quad 9(16.4) \quad 0.005^{*}$

$179(92.3) \quad 133(95.7) \quad 46(83.6)$

Drink coffee/strong tea $(n$, $\%)$

Yes

No

$3(1.6) \quad 3(2.2) \quad 0(0) \quad 0.272$

$191(98.4) \quad 136(98.0) \quad 55(100)$

Urinary frequency $(n, \%)$

No

Occasionally

Usually

Urine leaking $(n, \%)$

No

Occasionally

Usually

Difficult urination $(n, \%)$

Yes

No

$144(74.2) \quad 107(77.0) \quad 37(67.3) \quad 0.273$

$44(22.7) \quad 29(20.9) \quad 15(27.3)$

$6(3.1) \quad 3(2.2) \quad 3(5.5)$

$126(65.0) \quad 91(65.5) \quad 35(63.6) \quad 0.065$

$58(29.9) \quad 44(31.7) \quad 14(25.5)$

$10(5.2) \quad 4(2.9) \quad 6(10.9)$

$4(2.1) \quad 4(2.9) \quad 0(0) \quad 0.204$

$190(97.9) \quad 135(97.1) \quad 55(100)$

"Occasionally" refers to $\leq 3$ times a week. "Usually" refers to $>3$ times a week, ${ }^{*} P<0.05$

group III ( $P=0.014$ for "occasional/usual"). Notably, perineal tearing and perineal-lateral incision are not applicable to cesarean sections owing to the nature of the procedure.

Table 4 shows no significant difference being found for the LH measurements of $\mathrm{TrD}$, A-PD, and ALH, as well as for AUB among all women included in this study with and without DRA. However, Supplementary Table 1 shows a statistically significant increase only for resting state $\operatorname{TrD}(P=0.032)$ and ALH $(P=0.048)$ values between groups I and II. No statistically significant changes were present for any
Table 1 Differences in clinical data for women with or without DRA

\begin{tabular}{lcccc}
\hline Characteristics & All subjects & No DRA & DRA & $P$ value \\
\hline Number $(n)$ & 194 & 139 & 55 & \\
Maternal age (years) & $31.2 \pm 4.1$ & $30(28.8$ to 33$)$ & $30(28$ to 34.3$)$ & 0.762 \\
Height $(\mathrm{cm})$ & $163.5 \pm 4.9$ & $163(160$ to 167$)$ & $163(162.8$ to 168$)$ & 0.162 \\
Body weight $(\mathrm{kg})$ & $60.5(56.3-70)$ & $60(56$ to 70$)$ & $64(56.8$ to 70$)$ & 0.622 \\
BMI $\left(\mathrm{kg} / \mathrm{m}^{2}\right)$ & $23.1(21.0-25.4)$ & $23.0(21.0$ to 25.4$)$ & $23.4(20.9$ to 25.3$)$ & 0.985 \\
Delivery mode $(n, \%)$ & & & & $0.038^{*}$ \\
VD & & $93(73.2)$ & $28(26.8)$ & \\
CS & & $46(63.0)$ & $27(37.0)$ & \\
\hline
\end{tabular}

$B M I$ body mass index, $V D$ vaginal delivery, $C S$ cesarean section, $N o D R A$ without diastasis recti abdominis, $D R A$ diastasis recti abdominis, $* P<0.05$ 
Table 3 Clinical symptoms for the four groups of women

\begin{tabular}{|c|c|c|c|c|c|c|}
\hline \multirow[b]{2}{*}{ Variable } & \multicolumn{2}{|l|}{ Vaginal delivery } & \multirow[t]{2}{*}{$P$} & \multicolumn{2}{|l|}{ Cesarean delivery } & \multirow[t]{2}{*}{$P$} \\
\hline & Group I $(n=93)$ & Group II $(n=28)$ & & Group III $(n=46)$ & Group IV $(n=27)$ & \\
\hline Perineal-lateral incision $(n, \%)$ & & & 0.831 & & & \\
\hline Yes & $42(45.2)$ & $12(42.9)$ & & & & \\
\hline No & $51(54.8)$ & $16(57.1)$ & & & & \\
\hline Perineal tear $(n, \%)$ & & & $0.000^{*}$ & & & \\
\hline Yes & $6(6.5)$ & $9(32.1)$ & & & & \\
\hline No & 87 (93.6) & $19(67.9)$ & & & & \\
\hline Drink coffee/strong tea $(n, \%)$ & & & - & & & 0.180 \\
\hline Yes & $0(0)$ & $0(0)$ & & $3(6.5)$ & $0(0)$ & \\
\hline No & $93(100)$ & $28(100)$ & & $43(93.5)$ & $27(100)$ & \\
\hline Urinary frequency $(n, \%)$ & & & 0.433 & & & $0.014^{*}$ \\
\hline No & $65(69.9)$ & $18(64.3)$ & & $42(91.3)$ & $19(70.4)$ & \\
\hline Occasionally & $25(26.9)$ & $8(28.6)$ & & $4(8.7)$ & $7(25.9)$ & \\
\hline Usually & $3(3.2)$ & $2(7.1)$ & & $0(0)$ & $1(3.7)$ & \\
\hline Urine leaking $(n, \%)$ & & & 0.246 & & & 0.178 \\
\hline No & $52(55.9)$ & $15(53.6)$ & & $39(84.8)$ & $20(74.1)$ & \\
\hline Occasionally & $37(39.8)$ & $8(28.6)$ & & $7(15.2)$ & $6(22.2)$ & \\
\hline Usually & $4(4.3)$ & $5(17.9)$ & & $0(0)$ & $1(3.7)$ & \\
\hline Difficult urination $(n, \%)$ & & & 0.340 & & & 0.447 \\
\hline Yes & $3(3.2)$ & $0(0)$ & & $1(2.2)$ & $0(0)$ & \\
\hline No & $90(96.8)$ & $28(100)$ & & $45(97.8)$ & $27(100)$ & \\
\hline
\end{tabular}

Group I: no DRA and VD; group II: DRA and VD; group III: no DRA and CS; group IV: DRA and CS

parameters measured in groups III and IV, no matter whether the state was rest, squeeze, or Valsalva.

When comparing between groups I and III as well as groups II and IV in Supplementary Table 2, to determine the presence of significant differences based on delivery mode, such differences were found between groups I and III for $\operatorname{TrD}$ $(P=0.005)$ and ALH $(P=0.035)$ at rest in the form of decreases. This was also the case for groups II and IV (respectively $P=0.007$ and $P=0.021$ ) as well as for A-PD at rest $(P=0.006)$. Significant decreases in $\operatorname{TrD}(P=0.005)$, A-PD
Table 4 Measurements of the levator hiatus and angle of the bladder and urethra in women with or without DRA

\begin{tabular}{llccl}
\hline Measurement status & Measuring section & No DRA $(n=139)$ & DRA $(n=55)$ & $P$ \\
\hline Rest & TrD $(\mathrm{cm})$ & $4.1(3.8$ to 4.3$)$ & $4.0(3.7$ to 4.5$)$ & 0.626 \\
& A-PD $(\mathrm{cm})$ & $5.1(4.6$ to 5.5$)$ & $5.2(4.6$ to 5.6$)$ & 0.882 \\
& ALH $\left(\mathrm{cm}^{2}\right)$ & $13.6(11.9$ to 15.9$)$ & $14.5(11.9$ to 15.8$)$ & 0.487 \\
Squeeze & ABU $($ degrees $)$ & $117.3(106.5$ to 126.5$)$ & $115.7(104.8$ to 132.1$)$ & 0.632 \\
& TrD $(\mathrm{cm})$ & $3.8(3.5$ to 4.1$)$ & $3.8(3.5$ to 4.1$)$ & 0.939 \\
& A-PD $(\mathrm{cm})$ & $4.4(4.0$ to 4.8$)$ & $4.2(3.8$ to 4.7$)$ & 0.183 \\
& ALH $\left(\mathrm{cm}^{2}\right)$ & $11.2(9.7$ to 13.0$)$ & $10.8(9.8$ to 12.7$)$ & 0.629 \\
Valsalva & ABU $($ degrees $)$ & $116.1(108.9$ to 126.1$)$ & $117.0(107.3$ to 125.0$)$ & 0.665 \\
& TrD $\left(\mathrm{cm}^{2}\right)$ & $4.8(4.3$ to 5.4$)$ & $4.7(4.3$ to 5.3$)$ & 0.823 \\
& A-PD $\left(\mathrm{cm}^{2}\right)$ & $6.3(5.5$ to 7.1$)$ & $6.0(5.3$ to 7.0$)$ & 0.518 \\
& ALH $\left(\mathrm{cm}^{2}\right)$ & $22.9(18.2$ to 28.5$)$ & $22.2(16.9$ to 28.0$)$ & 0.829 \\
& ABU $($ degrees $)$ & $147.3(130.9$ to 160.4$)$ & $154.1(133.6$ to 166.1$)$ & 0.108 \\
\hline
\end{tabular}

$\operatorname{Tr} D$ transverse diameter of the levator hiatus at the level of the pubovaginalis; $A-P D$ antero-posterior diameter of the levator hiatus;

$A L H$ area of the levator hiatus; $A B U$ angle of the bladder and urethra; $* P<0.05$ 
$(P=0.001)$, and ALH $(P=0.005)$ were also found between groups II and IV under squeeze conditions. As for Valsalva, TrD decreased significantly, while A-PD increased significantly between groups II and IV (respectively $P=0.002$ and $P=0.008)$.

\section{Discussion}

Our study has revealed a number of interesting findings with respect to the occurrence of DRA as well as its connections to delivery mode, symptoms, and levator hiatus measurements. First, DRA more frequently occurs in CS patients compared to VD. It is also more associated with perineal tears overall, and particularly in VD patients. Both these findings could respectively result from the direction of the contractive forces from abdominal and uterine muscles; during vaginal delivery, these forces are directly oriented toward the infant within the uterus to ensure delivery. This leads to rectus abdominis muscle extrusion and increased intraperitoneal pressure, yielding DRA and perineal tears. CS patients, by contrast, have more prevalent associations between urinary frequency and DRA, possibly due to complications from surgery associated with cesarean section. With respect to levator hiatus measurements, such as $\operatorname{TrD}$, A-PD, ALH, and AUB, overall, the presence or absence of DRA has no significant impact. However, a microanalysis revealed that VD patients with DRA have significant differences in $\operatorname{TrD}$ and ALH at rest, as well as AUB at Valsalva, compared to those without DRA, which may stem from overstretching of the pelvic floor muscles, as well as bladder squeezing, during delivery. Furthermore, significant differences in TrD and ALH are seen when comparing VD and CS without DRA at rest. More differences are present between VD and CS with DRA for TrD, A-PD, and ALH at rest and squeeze states as well as for only $\operatorname{TrD}$ and A-PD at Valsalva. All these findings could stem from the levator hiatus and other abdominal muscles being less stretched during cesarean section compared to vaginal delivery, explaining the smaller dimensions for most of the aforementioned parameters ( $\mathrm{TrD}$, ALH, AUB, A-PD); coupled with possible scar formation at the diastasis rectus abdominis post-surgery, this may yield greater susceptibility to DRA.

PFD severely impacts quality of life in women and is heavily influenced by pregnancy and delivery [22] . Its relationship with DRA and PFD is a major topic of discussion among urologists, gynecologists, and obstetricians. The present study therefore factored various covariates into the analysis, including delivery mode, as well as interactions among POP, UI, and pelvic pain with DRA, which have been studied by other researchers $[4,10]$.

Because the rectus abdominis diameter is influenced by pelvic muscle and abdominal external oblique muscle contractions, it is no surprise that the occurrence of DRA was affected by the delivery mode [23]. Our findings showed that women with a history of cesarean section were more prone to develop DRA, where the CS group had an incidence rate of $37.0 \%$ versus $26.8 \%$ in the VD group. The overall incidence, as determined from the clinical data, was $28.4 \%$. All these discoveries were compatible with previous studies $[9,10]$, where the literature indicated a DRA rate of 35-39\% for CS patients and a lower rate among VD patients. It should be noted, however, that DRA occurrence during the pregnancy and post-partum periods can vary, though the latter typically entails a progressive post-delivery decrease in the rectus abdominis inner diameter, albeit without ever vanishing [24]. The lower occurrence of diastasis during vaginal delivery could be caused by the contractive muscle force of the abdominal muscle groups being concentrated inward, directly on the uterus, to ensure the delivery of the infant. This was simultaneously coupled with the concentric extrusion of the rectus abdominis muscle, thus decreasing its inner diameter. Overall, the results from the present study were consistent with similar ones, such as the study conducted by Qing Wang et al. [10].

Traditionally, DRA is expected to more easily occur among leaner women because of the protective effects bestowed by abdominal wall fat. However, our study found that the body weight among the DRA group was slightly higher than for those with no-DRA (Table 1). This may be the result of our data not including individuals with BMI > $30 \mathrm{~kg} / \mathrm{m}^{2}$, which is supported by the lack of any statistically significant difference in BMI among the women tested between the DRA and no DRA groups, as they were both around $23 \mathrm{~kg} / \mathrm{m}^{2}$.

Our data also showed that perineal tearing is more likely to occur in the DRA and VD group. Perineal tear was one of the risk factors identified for some diseases, and the question of whether DRA causes perineal tearing, or vice versa, is worth further study [25]. One possibility for the connection between vaginal delivery and perineal tearing could be the inward contraction of abdominal muscles, particularly uterine, during delivery. Upon reaching a certain level of intraperitoneal pressure, greater than what the muscles can bear, the intraabdominal pressure expands outwards to find a "weak spot" (the levator hiatus and perineal body [26]) in order to deliver the fetus, if it has not emerged yet. Under such circumstances, the abdominal-pelvic cavity is not ready for delivery, which might cause the rectus abdominis diastasis and perineal tear. This connection is also supported by lower linea alba stiffness in the DRA group, according to the literature [27].

The occurrence of certain lower urinary tract symptoms, such as urinary frequency and usually having urine leakage, is more prevalent in DRA than in no DRA patients in terms of patient percentages. However, no statistically-significant increases were present when accounting for all cases in both the urinary frequency and leakage categories. 
When the delivery mode is not considered, no statistically significant differences were present between DRA and no DRA patients. However, if delivery mode was accounted for, statistically significant differences were found between VD without DRA versus with DRA in regard to $\operatorname{TrD}$ and ALH at rest, as well as AUB at Valsalva. These differences were not found between CS patients with and without DRA, indicating that different delivery modes may affect levator hiatus measurements under DRA conditions. On the other hand, even without significant differences, most levator hiatus measurements for the DRA group are larger than for those with no DRA, possibly indicating an indirect effect between DRA and POP.

Our study was limited by the modest sample size, as well as observation biases, owing to the patients requiring strict and regular screening. Additionally, future studies would need to include pre-pregnancy information, as well as other childbirth-related factors, such as large birth weight babies [5], in order to exclude the confounding factors in relation to the occurrence of DRA and the delivery method. It is also worth noting that the follow-up stopped at 6 months postpartum but morphological changes related to pregnancy may continue to evolve well beyond that timeline. Therefore, some of these changes might have been temporary.

In conclusion, DRA and PFD are of clinical relevance, in that the occurrence of DRA varies with delivery mode, where women with cesarean section may be more susceptible to DRA. Furthermore, DRA patients were more likely to have urological symptoms and larger levator hiatus measurements.

Supplementary Information The online version contains supplementary material available at https://doi.org/10.1007/s00192-021-04783-1.

Acknowledgements We appreciate the statistical advice provided by Wenbo Tian of Sun Yat-sen University, as well as Wei Sun and Ning Dong of Daqing Oilfield General Hospital for their data collection. We thank Alina Yao for helping with the manuscript preparation and editing.

Authors' contributions P Tian: project development, data collection, statistical analysis, manuscript writing; DM Liu: data collection, manuscript writing; C Wang: manuscript revision; Y Gu: data collection, manuscript revision; GQ Du: Project development, manuscript revision; JW Tian: Project development, final approval of the manuscript.

Declarations Written informed consent was obtained from the patients for publication of the images in Urogynecology.

Conflict of Interest None.

Open Access This article is licensed under a Creative Commons Attribution 4.0 International License, which permits use, sharing, adaptation, distribution and reproduction in any medium or format, as long as you give appropriate credit to the original author(s) and the source, provide a link to the Creative Commons licence, and indicate if changes were made. The images or other third party material in this article are included in the article's Creative Commons licence, unless indicated otherwise in a credit line to the material. If material is not included in the article's Creative Commons licence and your intended use is not permitted by statutory regulation or exceeds the permitted use, you will need to obtain permission directly from the copyright holder. To view a copy of this licence, visit http://creativecommons.org/licenses/by/4.0/.

\section{References}

1. Spitznagle TM, Leong FC, Van Dillen LR. Prevalence of diastasis recti abdominis in a urogynecological patient population. Int Urogynecol J Pelvic Floor Dysfunct. 2007;18(3):321-8. https:// doi.org/10.1007/s00192-006-0143-5.

2. Gitta S, Magyar Z, Tardi P, et al. Prevalence, potential risk factors and sequelae of diastasis recti abdominis. Orv Hetil. 2017;158(12): 454-60. https://doi.org/10.1556/650.2017.30703.

3. Bo K, Hilde G, Tennfjord MK, Sperstad JB, Engh ME. Pelvic floor muscle function, pelvic floor dysfunction and diastasis recti abdominis: prospective cohort study. Neurourol Urodyn. 2017;36(3):716-21. https://doi.org/10.1002/nau.23005.

4. Sperstad JB, Tennfjord MK, Hilde G, Ellstrom-Engh M, Bo K. Diastasis recti abdominis during pregnancy and 12 months after childbirth: prevalence, risk factors and report of lumbopelvic pain. Br J Sports Med. 2016;50(17):1092-6. https://doi.org/10.1136/ bjsports-2016-096065.

5. Fernandes da Mota PG, Pascoal AG, Carita AI, Bo K. Prevalence and risk factors of diastasis recti abdominis from late pregnancy to 6 months postpartum, and relationship with lumbo-pelvic pain. Man Ther. 2015;20(1):200-5. https://doi.org/10.1016/j.math.2014.09. 002.

6. Mota P, Pascoal AG, Sancho F, Bo K. Test-retest and intrarater reliability of 2-dimensional ultrasound measurements of distance between rectus abdominis in women. J Orthop Sports Phys Ther. 2012;42(11):940-6. https://doi.org/10.2519/jospt.2012.4115.

7. Mota P, Pascoal AG, Sancho F, Carita AI, Bo K. Reliability of the inter-rectus distance measured by palpation. Comparison of palpation and ultrasound measurements. Man Ther. 2013;18(4):294-8. https://doi.org/10.1016/j.math.2012.10.013.

8. van de Water AT, Benjamin DR. Measurement methods to assess diastasis of the rectus abdominis muscle (DRAM): a systematic review of their measurement properties and meta-analytic reliability generalisation. Man Ther. 2016;21:41-53. https://doi.org/10.1016/ j.math.2015.09.013.

9. Toozs-Hobson P, Balmforth J, Cardozo L, Khullar V, Athanasiou $\mathrm{S}$. The effect of mode of delivery on pelvic floor functional anatomy. Int Urogynecol J Pelvic Floor Dysfunct. 2008;19(3):407-16. https://doi.org/10.1007/s00192-007-0455-0.

10. Wang Q, Yu X, Chen G, Sun X, Wang J. Does diastasis recti abdominis weaken pelvic floor function? A cross-sectional study. Int Urogynecol J. 2019;31(2):277-83. https://doi.org/10.1007/ s00192-019-04005-9.

11. Mulder FE, Shek KL, Dietz HP. What's a proper push? The Valsalva manoeuvre revisited. Aust N Z J Obstet Gynaecol. 2012;52(3):282-5. https://doi.org/10.1111/j.1479-828X.2012. 01446.x.

12. Machin D, Campbell M, Fayers P, Pinol A. Sample size tables for clinical studies. 2nd edition. Malden: Blackwell Science; 1997.

13. Zar HJ. Biostatistical analysis. Second edition edn. Englewood Cliffs: Prentice-Hall; 1984.

14. Al-Sunduqchi (1990) Determining the Appropriate Sample Size for Inferences Based on the Wilcoxon Statistics. Dissertation, University of Wyoming. 
15. Mota P, Pascoal AG, Carita AI, Bo K. Normal width of the interrecti distance in pregnant and postpartum primiparous women. Musculoskelet Sci Pract. 2018;35:34-7. https://doi.org/10.1016/j. msksp.2018.02.004.

16. Reinpold W, Kockerling F, Bittner R, et al. Classification of rectus diastasis-a proposal by the German Hernia Society (DHG) and the International Endohernia Society (IEHS). Front Surg. 2019;6(1): 10.3389/fsurg.2019.00001.

17. Beer GM, Schuster A, Seifert B, et al. The normal width of the linea alba in nulliparous women. Clin Anat. 2009;22(6):706-11. https:// doi.org/10.1002/ca.20836.

18. Dalinjong PA, Laar AS. The national health insurance scheme: perceptions and experiences of health care providers and clients in two districts of Ghana. Health Economics Rev. 2012;2:13. https:// doi.org/10.1186/2191-1991-2-13.

19. Dietz HP, Rojas RG, Shek KL. Postprocessing of pelvic floor ultrasound data: how repeatable is it? Aust N Z J Obstet Gynaecol. 2014;54(6):553-7. https://doi.org/10.1111/ajo.12250.

20. Haylen BT, Maher CF, Barber MD, et al. An International Urogynecological Association (IUGA)/International Continence Society (ICS) joint report on the terminology for female pelvic organ prolapse (POP). Int Urogynecol J. 2016;27(4):655-84. https://doi.org/10.1007/s00192-016-3003-y.

21. Braekken IH, Majida M, Ellstrom-Engh M, et al. Test-retest and intra-observer repeatability of two-, three- and four-dimensional perineal ultrasound of pelvic floor muscle anatomy and function. Int Urogynecol J Pelvic Floor Dysfunct. 2008;19(2):227-35. https://doi.org/10.1007/s00192-007-0408-7.

22. Wilson PD, Herbison RM, Herbison GP. Obstetric practice and the prevalence of urinary incontinence three months after delivery. Br J
Obstet Gynaecol. 1996;103(2):154-61. https://doi.org/10.1111/j. 1471-0528.1996.tb09668.x.

23. Theodorsen NM, Strand LI, Bo K. Effect of pelvic floor and transversus abdominis muscle contraction on inter-rectus distance in postpartum women: a cross-sectional experimental study. Physiotherapy. 2018. https://doi.org/10.1016/j.physio.2018.08. 009 .

24. Boissonnault JS, Blaschak MJ. Incidence of diastasis recti abdominis during the childbearing year. Phys Ther. 1988;68(7): 1082-6. https://doi.org/10.1093/ptj/68.7.1082.

25. Ngunyi YL, Halle-Ekane G, Tendongfor $\mathrm{N}$ et al. Determinants and aetiologies of postpartum pyrexia; a retrospective analysis in a tertiary health facility in the Littoral Region of Cameroon. BMC Pregnancy and Childbirth.2020; 20 (1).https://doi.org/10.1186/ s12884-020-02867-2.

26. Wu Y, Dabhoiwala NF, Hagoort J, et al. Architectural differences in the anterior and middle compartments of the pelvic floor of youngadult and postmenopausal females. J Anat. 2017;230(5):651-63. https://doi.org/10.1111/joa.12598.

27. Beamish N, Green N, Nieuwold E, McLean L. differences in linea alba stiffness and linea alba distortion between women with and without diastasis recti abdominis: the impact of measurement site and task. J Orthop Sports Phys Ther. 2019:1-30.https://doi.org/10. 2519/jospt.2019.8543

Publisher's note Springer Nature remains neutral with regard to jurisdictional claims in published maps and institutional affiliations. 